\title{
The Interplay of Nuclear and Atomic Physics \\ in the Synthesis of the Elements
}

LBL -27392

DE89 016305

Eric B. Norman

Nuclear Science Division, I zwrence Berkeley Laboratory, and

Center for Particle Astrophys.cs University of California Berkeley, California 94720 U. S. A.

Abstraci

In many astronomical environments, physical conditions are so extreme that matter is almost completely ionized. The absence of bound atomic electrons can dramatically alter the decay rates of a number of radioactive nuclei. Several examples of this interplay of nuclear and atomic physics relevant to the synthesis of the chemical elements are described.

\section{DISCLAIMER}

This report was prepared as an account of work sponsored by an asency of the Unised States Government. Neither the United States Government nor any agency thereof, nor any of their emptoyees, makes any warranty, express or implied, or assumes any legal liability or responsibility for the accuracy, completeness, or usefulness of any information, apparatus, product, or process disclosed, or represents that its use would not infringe privately owned rights. Reference herein to any specific commercial product, process, or service by trade name, trademark, manufacturer, or otherwise does not necessarily constitute or imply its endorsement, recommendation, or favoring by the United States Government or any agency thereof. The views and opinions of authors expressed herein do nut necessarily state or reflect those of the United States Government or any agency thereof. 


\section{Introduction}

Under the extreme physical conditions found in stellar interiors and other astronomical environments, matter is essentially fully ionized. The absence of atomic electrons produces effects that are of importance in stellar nucleosynthesis and in cosmic ray physics. Some species of astrophysical interest are radioactive nuclei which, under terrestrial conditions, decay by the capture of a bound atomic electron. At the high temperatures found in stellar interiors, such nuclei can only decay via electron capture from the continuum. High energy cosmic rays are fully stripped of their atomic electrons in passing through the interstellar medium. Thus, because there are no bound electrons to be captured, dramatic decreases in the decay rates occur for such nuclei. For some species, the result is that they become "stable" while for others, terrestrially unobserved $\beta^{*}$ or $\beta^{+}$decays will determine their decay rates. Another effect that results from the absence of atomic electrons is bound state $\beta$ decay. In this process, the beta particle is emitted directly into a previously unoccupied bound atomic orbital. For decays with low endpoint energies, the increase in decay energy provided by the atomic binding energy can lead to large increases in the nuclear decay rate. Effects such as these illustrate the interplay between nuclear and atomic physics in the synthesis of the elements. In this talk, I will discuas several examples of current problems in nuclear astrophysics in which such processes are important.

\section{${ }^{7} \mathrm{Be}_{\mathrm{Q}}$}

The fusion of hydrogen into helium in the Sun is believed to occur via a sequence of nuclear reactions known as the p-p chain. The only direct evidence 
for this is provided by the observation of solar neutrinos that are produced during these reactions. The experimental result of Davis [1] and his collaborators is about a factor of three below that predicted by Bahcall [1] and others. This discrepancy is known as the "Solar Neutrino Problem." The Davis experiment is a

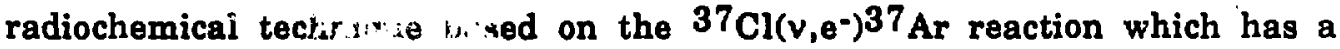
threshold energy ui $314 ; \therefore$. Thus it is mainly sensitive to the rare but high energy neutrinos from the $\beta$-decay of ${ }^{8} \mathrm{~B}$ which is produced via the ${ }^{7} \mathrm{Be}(\mathrm{p}, y)$ reaction. This proton capture reaction must compete with the electron capture decay of ${ }^{7} \mathrm{Be}$. In the laboratory, neutral ${ }^{7} \mathrm{Be}$ atoms decay with a half-life of 53 days. However, the K-electron binding energy of $\mathrm{Be}$ is only $0.22 \mathrm{keV}$, while the mean thermal energy, $\mathrm{kT}=1.3 \mathrm{keV}$ at the center of the Sun. Therefore, in the inner regions of the Sun where the fusion occurs, ${ }^{7} \mathrm{Be}$ is fully ionized. Thus, electron capture proceed by capture from the continuum. This process has been investigated by Bahcall and Moeller [2] who have estimated that averaged over the inner $10 \%$ of the Sun, where over $95 \%$ of the ${ }^{8} \mathrm{~B}$ is produced, the effective half-life of ${ }^{7 \mathrm{Be}}$ is approximately 70 days. This is about a $40 \%$ increase over the laboratory value and leads to a correspondingly higher ${ }^{8} \mathrm{~B}$ production rate than would be inferred from the laboratory half life. This effect is properly taken into account in estimates of the solar neutrino flux, but illustrates the connection between nuclear and atomic effects in nucleosynthesis.

\section{Mn}

In the laboratory, neutral $54 \mathrm{Mn}$ atoms decay with a 312 day half-life via an allowed electron capture transition to the $835 \mathrm{keV}$ level in ${ }^{54} \mathrm{Cr}$. In the cosmic rays, ${ }^{54} \mathrm{Mn}$ is believed to be produced through spallation of primary iron nuclei by interstellar hydrogen and would be stripped of all its atomic electrons, While this would prevent its decay by electron capture, as can be seen from Figure 1, it is 
energetically possible for it to decay via second forbidden unique transitions to the ground states of ${ }^{54} \mathrm{Cr}$ and $54 \mathrm{Fe}$ by positron or negatron emission, respectively. Because of the expected long $\beta^{+}$and $\beta$ - lifetimes, $54 \mathrm{Mn}$ has been proposed as a cosmic-ray chronometer [3]. More recently, the presence of ${ }^{54} \mathrm{Mn}$ in the cosmic rays has been experimentally confirmed [4], with a half-life estimated to be ( 1 to 2 ) $\times 10^{6}$ years in order to explain the measured abundance as a function of cosmicray energy [5].

While the dominant decay mode for fully ionized $54 \mathrm{Mn}$ is expected to be $\beta$ decay, it is extremely hard to experimentally isolate this small branch in the laboratory. Thus, the initial studies of Sur et al. [6] searched for the even smaller, but easier to detect, $\beta^{+}$decay branch. In this experiment a shemically purified $7.3 \mu \mathrm{Ci} 54 \mathrm{Mn}$ source was mounted in front of a two-element silicon surface-barrier detector telescope to detect the emitted positrons. The source and telescope were mounted at the center of an annular $30 \mathrm{~cm} \times 30 \mathrm{~cm} \mathrm{NaI}$ detector that was optically divided into two halves. The back-to-back 511-keV $\gamma$ rays from positrons annihilating in the back element of the telescope were detected in these two halves of the annular detector. A 4-fold coincidence between the telescope elements and each half of the annular $\mathrm{NaI}$ detector was required in the electronic hardware trigger. The efficiency of the apparatus for detecting positrons from the decay of $54 \mathrm{Mn}$ was measured by detecting positrons from a $65 \mathrm{Zn}$ source mounted in the same geometry as the $54 \mathrm{Mn}$.

The candidate positron spectrum was extracted by projecting out the summed telescope energy spectrum in coincidence with $511 \mathrm{keV} \gamma$ rays detected in both halves of the annular $\mathrm{NaI}$ detector within the prompt timing requirement for all detectors. Background spectra were extracted by projecting the summed telescope energy spectrum in coincidence with combinations of gates set above and below $511 \mathrm{keV}$ in both halves of the $\mathrm{NaI}$ annulus. During a total running time of 360.4 
hours, $208 \pm 14.4$ events were observed in the first $355 \mathrm{keV}$ of the summed telescope energy spectrum in the $511-511 \mathrm{keV}$ gate of interest. The average number of events in all the background gates was $213 \pm 5.6$. Thus the $1 \sigma$ upper limit on the number of $54 \mathrm{Mn}$ positrons detected was 15.5 . Using the experimentally determined efficiency of $0.10 \%$ for detecting positrons, this results in an upper limit of $4.4 \times 10^{-8}$ for the $\beta+$ decay branching ratio of $54 \mathrm{Mn}$, which corresponds to a lower limit of $2.0 \times 10^{7}$ years for the partial half-life of this decay channel. This result clearly rules out the possibility that $\beta+$ is the dominant decay mode for the $54 \mathrm{Mn}$ nuclei found in cosmic rays. Using the accepted value of $1377 \mathrm{keV}$ for the mass difference between ${ }^{54} \mathrm{Mn}$ and ${ }^{54} \mathrm{Cr}$, a lower limit of 13.3 was established for the $\log \mathrm{ft}$ value of this transition. Uaing this $\log \mathrm{ft}$ value for the $\beta^{-}$decay branch with $697 \mathrm{keV}$ of available energy, a lower limit of $4 \times 10^{4}$ years was obtained for the partial half-life of this decay mode. This value is about 25 times shorter than the estimated half-life of $54 \mathrm{Mn}$ in cosmic rays and thus consistent with $\beta-$ as the dominant channel for this decay. Experiments to measure the $\beta$ - decay rate of $54 \mathrm{Mn}$ are now in progress.

\section{${ }^{176} \mathrm{~T}_{\mathrm{du}}$}

${ }^{176} \mathrm{Lu}$ is one of the few naturally occurring radioactive nuclides that have survived from the era of nucleosynthesis. As can be seen from Figure 2, it is produced only in the slow neutron capture process (8-process) and thus was suggested by Audouze, Fowler and Schramm [7] and independently by Amould [8] to be a possible 8-process chronometer. The use of $176 \mathrm{Lu}$ as such a clock assumes that its decay rate is unaffected by environmental factors. Under terrestrial conditions, this is an extremely good approximation. However, the 
nuclear structure of $176 \mathrm{Lu}$ suggests that this is not the case in the heliumburning zones of red giant stars where the s-process is believed to occur.

The $\mathrm{J}^{\pi}=7^{-}$ground state, ${ }^{176} \mathrm{Lug}$, beta decays to ${ }^{176} \mathrm{Hf}$ with a half life of $3.6 \mathrm{x}$ $10^{10}$ years. The first excited state, ${ }^{176} \mathrm{Lu} m$, is a $\mathrm{J} \pi=1^{-1}$ level at approximately 123 $\mathrm{keV}$ that beta decays to $176 \mathrm{Hf}$ with a 3.7 hour half life but does not decay to 176 Lus. If these two levels could come into thermal equilibrium, then the effective half life of this nucleus would be very temperature dependent and could be orders of magnitude shorter than the laboratory half life of $176 \mathrm{Lug}$. Among the possible mechanism, that would allow $176 \mathrm{Lug}, \mathrm{m}$ to "communicate" are photoexcitation, inelantic neutron scattering, Coulomb excitation, and positron annihilation excitation. As illustrated in Figure 3, all of these processes undoubtedly involve transitions from ${ }^{176} \mathrm{Lug}, \mathrm{m}$ to some higher lying levels of intermediate spin which subsequently decay to $176 \mathrm{Lu} \mathbf{m}, \mathrm{g}$.

The interesting process of positron annihilation excitation of $176 \mathrm{Lu}$ has been observed. [9] In the laboratory, a positron annihilates with a bound atomic electron (most probably a K-shell electron). Then, rather than the usual twophoton annihilation radiation being emitted, it is likely that a single real annihilation photon is emitted and a virtual photon is absorbed by the nucleus producing a nuclear excited state. [10] A possible Feynman diagram of this process for the conversion of $176 \mathrm{Lug}$ to $176 \mathrm{Lum}$ is shown in Figure 4. The laboratory cross section for this phenomenon is $26 \pm 9 \mu \mathrm{b}$ [9] which is nearly a thousand times larger than the photoactivation cross section measured with ${ }^{60}$ Co $\gamma$ rays.[11] Thus given a sufficiently large number of positrons, this process could be a very effective ${ }^{176} \mathrm{Lug}$ destruction mechanism. The effects of this process under s-process conditions have been studied by Norman et al. [11] who found that positron annihilation excitation alone guarantees that $176 \mathrm{Lug}, \mathrm{m}$ are in thermal equilibrium for temperatures above $3.5 \times 10^{8} \mathrm{~K}$. 
Until recently, no excited states in ${ }^{176} \mathrm{Lu}$ had been observed that would allow this sort of "communication" between $176 \mathrm{Lug}, \mathrm{m}$. To address this issue, Sur et al. [12] used the ${ }^{176} \mathrm{Yb}(\mathrm{p}, \mathrm{n}){ }^{176} \mathrm{Lu}$ reaction and the High Energy Resolution Array of 20 Compton-suppressed germanium detectors to collect gamma-ray singles and gamma-gamma coincidence data. A level at $838.5 \mathrm{keV}$ was found to decay to both $176 \mathrm{Lu} 8$ and $176 \mathrm{Lum}$. The lifetime of this level was measured to be less than 10 nsec. With this information and the principle of detailed balance, the rate for photoexcitation of $176 \mathrm{Lu} 8$ to the $838.5-\mathrm{keV}$ level was calculated as a function of temperature. The results of these calculations show that photoexcitation alone guarantees that ${ }^{176} \mathrm{Lug}, \mathrm{m}$ are in thermal equilibrium for temperatures above 2.9 $x 10^{8} \mathrm{~K}$. The effective half life of this nucleus under such conditions is less than one year. The conclusion that can be drawn from all of this work is that the effective half life of ${ }^{176} \mathrm{Lu}$ is very temperature dependent anci that as a result, the abundance of $176 \mathrm{Lu}$ that emerges from an s-piocess environment is a very sensitive function of the thermal history that it has experienced. Thus ${ }^{176} \mathrm{Lu}$ is not a reliable chronometer.

\section{${ }^{187} \mathrm{Re}$}

As can be seen in Figure 2, the long-lived isotope ${ }^{187_{\text {Re }}}\left(t_{1 / 2}=4.3 \times 10^{10}\right.$ years $)$ is produced only in the r-process. It was therefore suggested as a potential $r$ process chronometer. [13] Again, however, there are nuclear and atomic physics complications which compromise the usefulness of this clock.

In the laboratory, a neutral ${ }^{187_{R}}$ atom decays to a neutral $187^{0}$ s atom with a $\beta$-decay endpoint energy of $2.6 \mathrm{keV}$. This energy is actually less than the $14 \mathrm{keV}$ difference in atomic electron binding energies between Re and Os. Thus, while a neutral ${ }^{187} \mathrm{Re}$ atom is more massive than a neutral $187 \mathrm{Os}$ atom, the situation is 
reversed when one considers the bare nuclei. As discussed previously, at the high temperatures required for both the s- and r-processes, all matter is nearly fully ionized. Thus one must consider the possibilities of the continuum electroncapture decay of $187 \mathrm{Os}$ to ${ }^{187_{\mathrm{Re}}}$ and the bound-state $\beta$-decay of ${ }^{187^{2} \mathrm{Re}}$ into previously unoccupied atomic orbitals.

The effects of these processes have been studied theoretically by Yokoi, Takahashi, and Amould. [14,15] The increased decay energy provided by the bound-state decay of fully ionized ${ }^{187}$ Re allows transitions to the $9.75-\mathrm{keV}$ first excited state of $187 \mathrm{O}$ and changes the $\beta$ - decay rate from $4.3 \times 10^{10}$ years to approximately 10 years! The bound state $\beta$ decay of any nucleus has yet to be observed in the laboratory, and its detection offers a real challenge to the experimentalist. Based on the calculated half-life of Takahashi and Yokoi, one can estimate that if one could "hold" $10^{6}$ fully ionized ${ }^{187}$ Re atoms for 1 day, then about 200 bound state $\beta$ decays would occur resulting in the production of that number of 187 os ions. A possible way to produce the fully ionized $187_{\text {Re and to }}$ detect this very small number of 187 Os ions would be to use the electron beam ion trap (EBIT) technique described by Levine. [16]

\section{Conclusions and Acknowledgements}

The four examples I have chosen illustrate some of the connections between nuclear and atomic physics relevant to understanding the synthesis of the chemical elements. There are a number of other nuclei of astrophysical interest for which similar effects are important. As a result, there are many challenging experiments yet to be done.

The experiments described in this talk involved the efforts of a number of different people. My collaborators on these projects have been K. T. Lesko, B. Sur, R. M. Larimer, E. Browne, K. R. Vogel, E. P. Horch. R. M. Diamond, F. S. 
Stephens, M. A. Deleplanque, C. Beausang, T. Bertram, S. E. Kellogg, S. Gil, and P. Wong. This work was supported by the Director, Office of Energy Research, Office of High inergy and Nuclear Physics, Nuclear Physics Division of the U. S. Department of Energy under Contract No. DE-AC03-76SF00098. 


\section{References}

1. J. N. Bahcall and R. Davis, in: Essays in Nuclear Astrophysics, eds., C. A. Barnes, D. D. Clayton, and D. N. Schramm (Cambridge Univ. Press, Cambridge, 1982) p. 243.

2. J. N. Bahcall and C. P. Moeller, Attrophys. Journ. 155 (1969) 511 .

3. M. Casse, Astrophys. Journ. 180, (1973) 623.

4. G. Tarle, S. P. Ahlen and B. G. Cartwright, Astrophys. Journ. 230, (1979) 607.

5. L. Koch et al., Astron.and Astrophys. 102, (1981) L9.

6. B. Sur et al. Phys. Rev. C 39 (1989) xux .

7. J. Audouze, W. A. Fowler, and D. N. Schramm, Nature 238 (1972) 8.

8. M. Annould, Astron. Astrophys. 22 (1973) 311 .

9. Y. Watanabe, T. Mukoyama, and R. Katano, Phys. Rev. C 23 (1981) 695.

10. R. S. Raghavan and A. P. Mills, Phys. Rev. C 24 (1981) 1814.

11. E. B. Norman et al., Astrophys. Journ. 291 (1985) 834.

12. B. Sur et al., Bull. Am. Phys. Soc. 34 (1989) $x \times x x$.

13. D. D. Clayton, Astrophys. Journ 139 (1964) 637.

14. K. Yokoi, K. Takahashi, and M. Amould, Astron. Astrophys. 117 (1983) 65.

15. K. Takahashi and K. Yokoi, Nucl. Phys. A 404 (1983) 578.

16. M. A. Levine, this conference. 


\section{Fioure Captions}

1. Decay scheme is $54 \mathrm{Mn}$.

2. Expected paths of the slow (s) and rapid $(r)$ neutron-capture processes near $A=180$. Stable (or very long-lived) nuclei are indicated by shaded triangles.

3. Partial level scheme of ${ }^{176} \mathrm{Lu}$ and the decay schemes of ${ }^{176} \mathrm{Lu}$ and ${ }^{176} \mathrm{Lum}$. The possibility of exciting the long-lived 176Lut to a higher lying state of intermediate spin which subsequently decays to ${ }^{176} \mathrm{Lum}$ is shown schematically.

4. A possible Feynman diagram for the process of positron annihilationexcitation $0^{\wedge}{ }^{176} \mathrm{Lu}$ to a higher-lying state of intermediate spin that subsequently decays to ${ }^{176} \mathrm{Lum}$. 


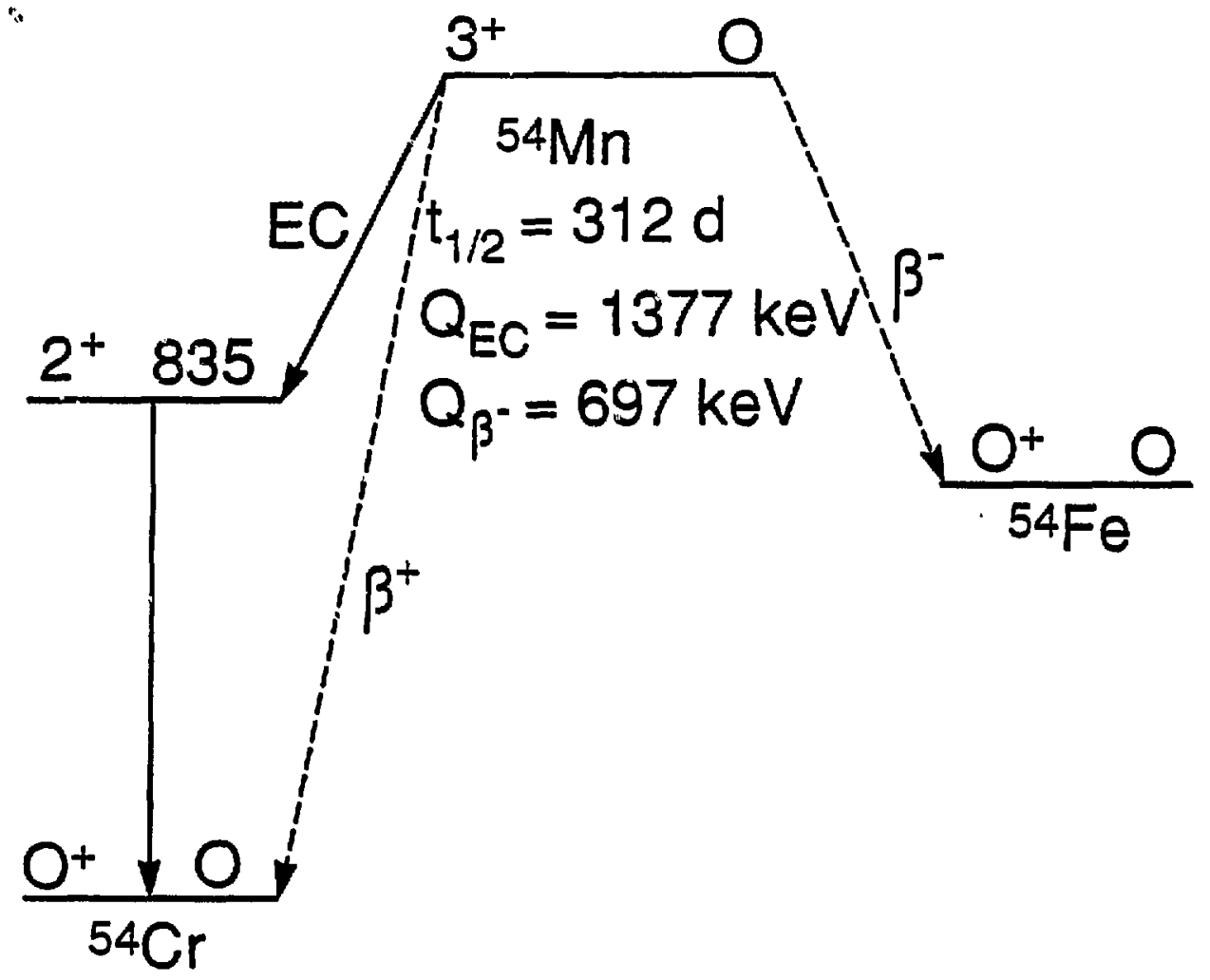

XBL 877-10265

Figure 1 


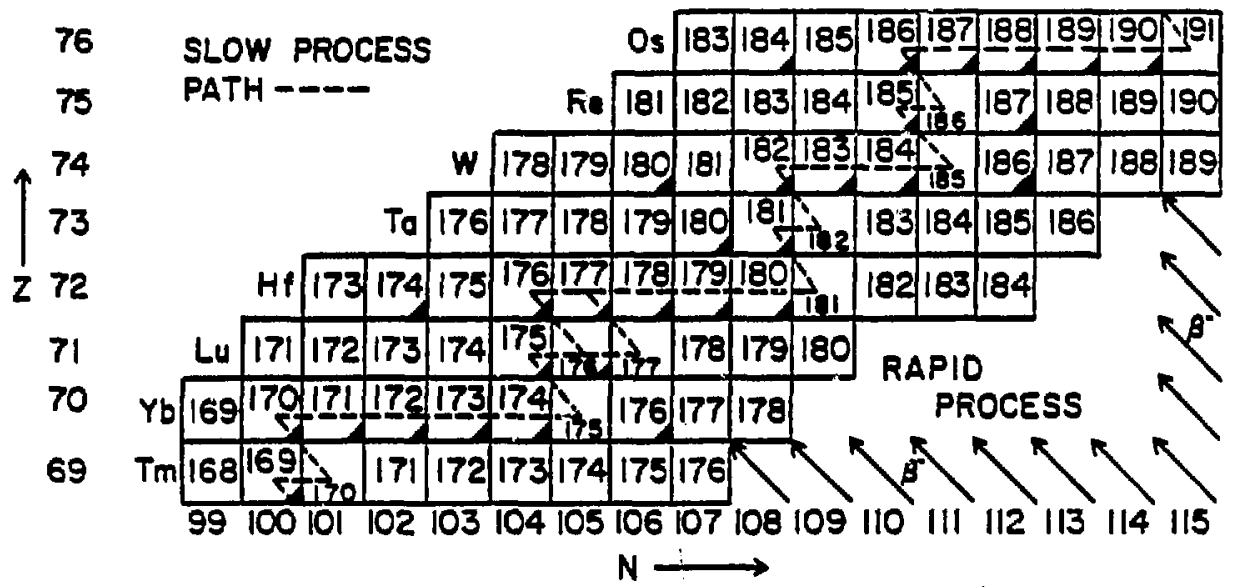

XBI 892-527

Figure 2 


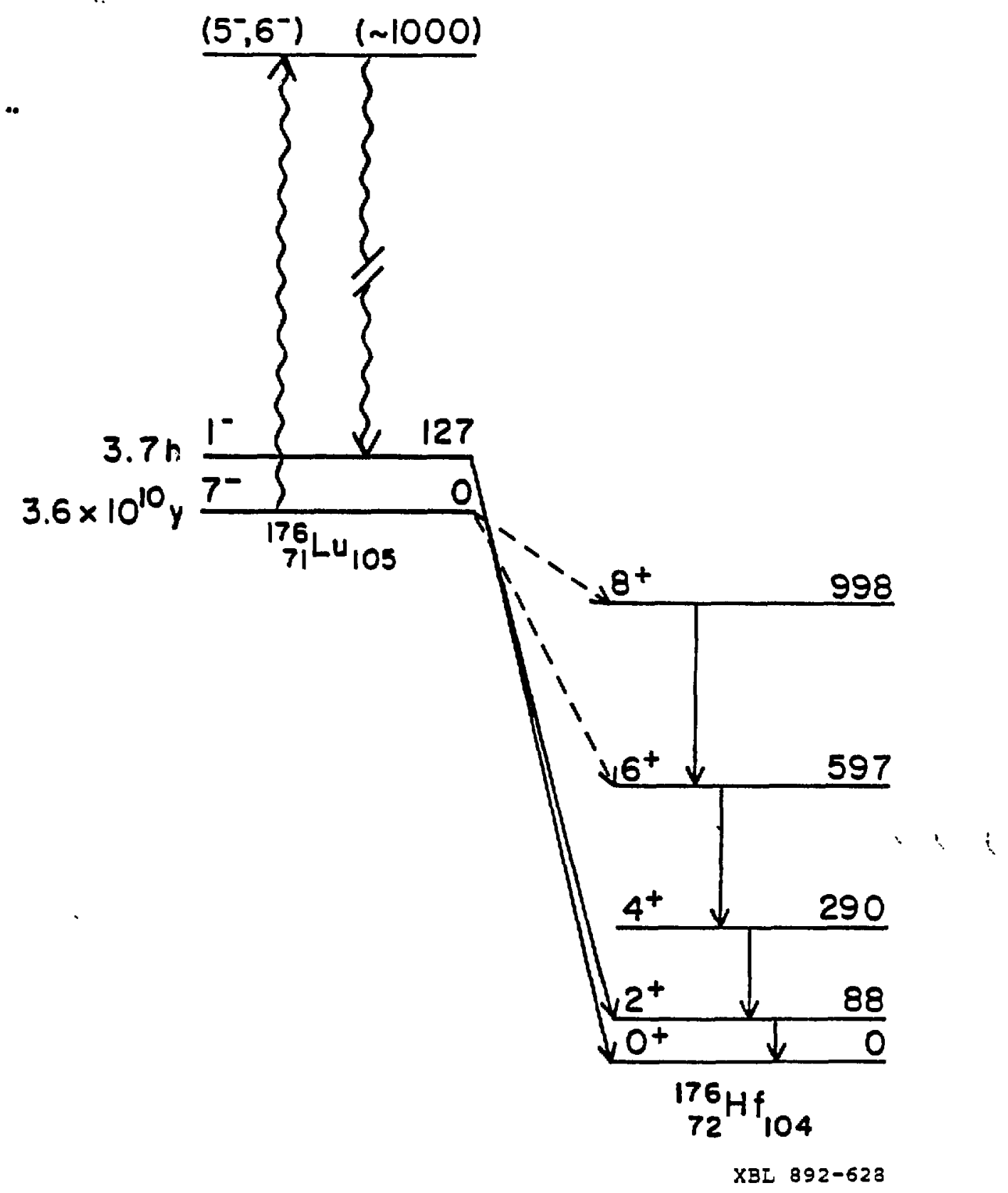

Figure 3 


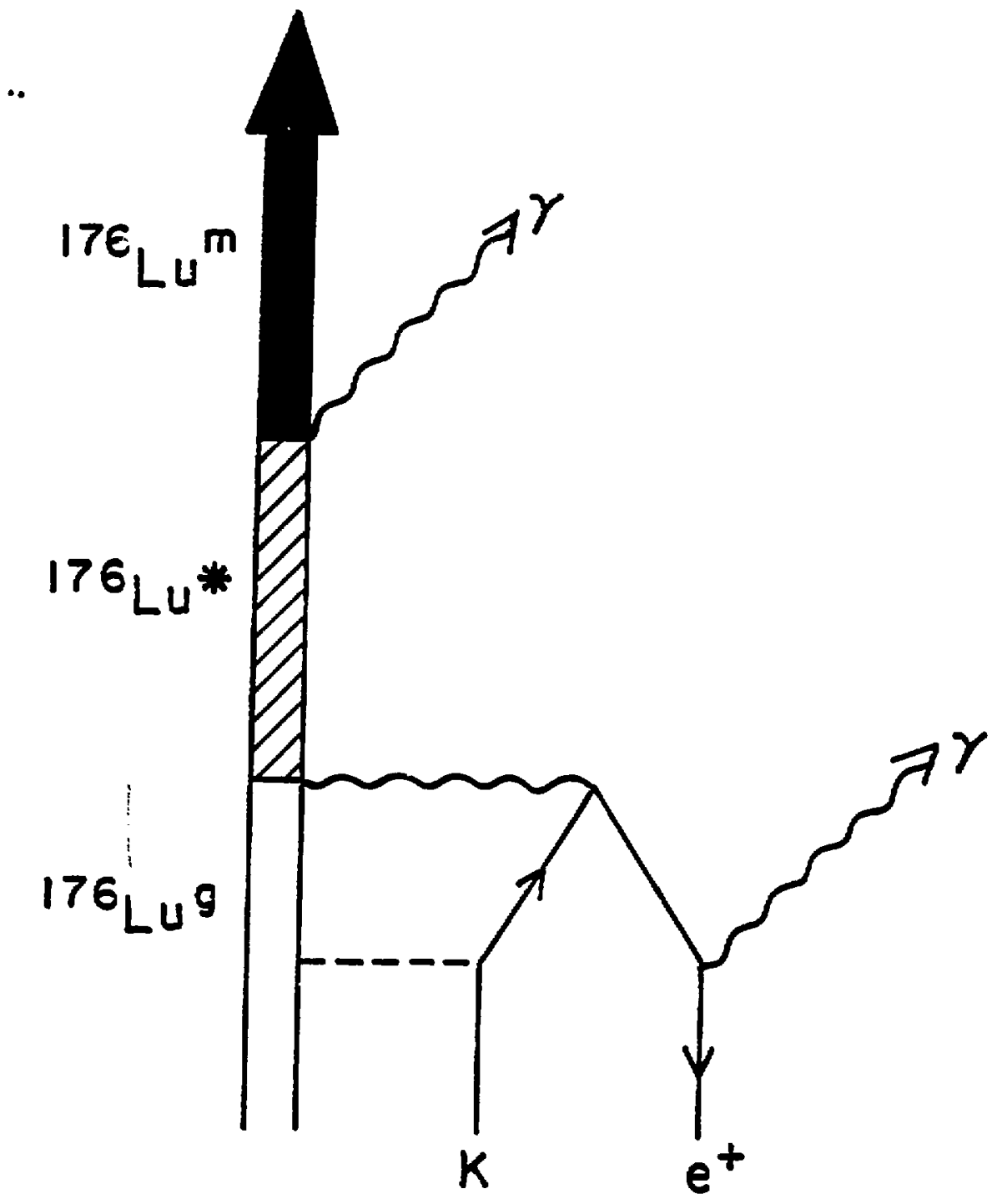

XBI $892-629$

Figure 4 